\title{
Accurate Mass Determination of Short-Lived Isotopes by a Tandem Penning-Trap Mass Spectrometer
}

\author{
H. Stolzenberg, St. Becker, G. Bollen, F. Kern, H.-J. Kluge, Th. Otto, G. Savard, and L. Schweikhard \\ Institut für Physik, Universität Mainz, D-6500 Mainz, Federal Republic of Germany \\ G. Audi \\ Centre de Spectrométrie Nucleaire et de Spectrometrie de Masse, Laboratoire René Bernas, \\ Bâtiment 108, F-91405 Orsay, France \\ R. B. Moore \\ Foster Radiation Laboratory, McGill University, Montreal, Québec, Canada H3A 2B2 \\ and The ISOLDE Collaboration, Division EP, CERN, CH-1211 Geneva, Switzerland
}

(Received 30 July 1990)

\begin{abstract}
A mass spectrometer consisting of two Penning traps has been set up for short-lived isotopes at the on-line mass separator ISOLDE at CERN. The ion beam is collected and cooled in the first trap. After delivery to the second trap, high-accuracy direct mass measurements are made by determining the cyclotron frequency of the stored ions. Measurements have been performed for ${ }^{118} \mathrm{Cs}-{ }^{137} \mathrm{Cs}$. A resolving power of over $10^{6}$ and an accuracy of $1.4 \times 10^{-7}$ have been achieved, corresponding to about $20 \mathrm{keV}$.

PACS numbers: $21.10 . \mathrm{Dr}, 07.75 .+\mathrm{h}, 27.60 .+\mathrm{j}$
\end{abstract}

There has been a decade-long effort to determine atomic masses ever more precisely and, surprisingly, a persistent demand to do it even better. This is because the atomic mass is a gross property which embodies all the effects of the interplaying forces in the nucleus.

Today, the nuclear binding energy of stable isotopes is generally known with an accuracy of the order of $\mathrm{keV} .{ }^{1}$ For unstable nuclei, the errors in mass determination increase rapidly for isotopes further from the valley of $\beta$ stability. Yet, it is the atomic masses of those short-lived isotopes with unusual $Z / N$ ratios that provide stringent tests of nuclear models, enable the discovery of new phenomena such as nuclear shape transitions, or are cornerstones in the interpretation of unexpected results such as the increased matter radius of ${ }^{11} \mathrm{Li}^{2}$ Furthermore, atomic masses are needed as input data for mass formulas which allow extrapolation to the masses of even more exotic isotopes.

The masses of most of the radioactive isotopes have been provided by nuclear reactions or the determination of $Q$ values in the nuclear decay. A major step forward was taken in the 1970s when magnetic spectrometers were put on-line with accelerators so as to measure the masses directly. ${ }^{3}$ During recent years the demand for precise masses of short-lived isotopes, or exotic particles, has prompted the development of new techniques for direct mass spectrometry. A general feature of these new devices is an evolution from measurements of voltage ratios to time and frequency. Time-of-flight mass spectrometry is performed by the facilities TOFI at Los Alamos ${ }^{4}$ and SPEG at GANIL, ${ }^{5}$ and is planned at ESR at GSI. ${ }^{6,7}$ Cyclotron resonance is another powerful technique first demonstrated for light masses ${ }^{8,9}$ and currently used at the Low Energy Antiproton Ring at CERN for a determination of the mass of the antiproton ${ }^{10,11}$ and at the on-line isotope separator ISOLDE at CERN for the measurement of masses of short-lived isotopes. ${ }^{12}$ The present Letter reports on the performance of the ISOLDE tandem Penning-trap mass spectrometer and the results obtained on neutron-deficient Cs isotopes.

The principle of the experiment is the determination of the cyclotron frequency $\omega_{c}=(q / m) B$ of an ion (mass $m$, charge $q$ ) stored in a Penning trap. The superimposed homogeneous magnetic field $B$ and the electric quadrupole field in a Penning trap confine the ion to a precessing circular motion with an axial oscillation. The precessional frequency, called the magnetron frequency $\omega_{-}$, is usually slow compared to the circular or cyclotron frequency $\omega_{+}{ }^{13}$ For a perfect Penning trap the sum of these two frequencies equals the cyclotron frequency of the ion in the absence of an electric field,

$$
\omega_{c}=2 \pi v_{c}=(q / m) B=\omega_{+}+\omega_{-} .
$$

Therefore the measurement of this sum frequency allows the direct determination of the mass $m$ of the stored ions. To determine this frequency the stored ions are excited by a radio-frequency (RF) field ${ }^{14}$ and the resonance is detected via time of flight. ${ }^{8}$ Because of the trap confinement, the RF field can be applied for an extended period, leading to a very high Fourier-limited resolving power.

It has been shown in Ref. 14 that an azimuthal quadrupole field at the sum frequency $v_{c}$ couples the cyclotron and magnetron motion leading to a periodic conversion of the one into the other. By proper choice of RF amplitude and excitation time as well as initial condi- 
tions on the ion motion, this results in a conversion from a pure magnetron into a pure cyclotron motion and in a large increase of radial energy.

The experimental setup shown in Fig. 1 consists of two Penning traps in tandem. The first trap, in an electromagnet $(B=0.7 \mathrm{~T})$, is for accumulating and cooling the continuous ion beam delivered by ISOLDE. The second high-precision trap (characteristic trap dimension of $10 \mathrm{~mm}$ ), for the actual measurements, is in a superconducting magnet $(B=5.7 \mathrm{~T})$. More details of the mechanical setup can be found in Ref. 12 .

For the first trap the $60-\mathrm{keV}$ ISOLDE beam is accumulated on a $\mathrm{Re}$ foil. The implanted radioactive sample is released and surface ionized by heating the foil. The ions are captured in the trap by collisions with He buffer gas ( $p_{\mathrm{He}}=10^{-3}-10^{-4}$ mbar). They are centered by a new sideband cooling technique based on the application of an azimuthal RF quadrupole field at $v_{\mathrm{RF}}=v_{c}$. As discussed above, such a RF excitation leads to the average radii of the magnetron and cyclotron motions being equal. Since the buffer-gas collisions cool the cyclotron motion along with the axial motion, a reduction of the magnetron motion is also obtained and the ions are centered in the trap. This process is mass selective and has been proven to remove all isotopic impurities.

After cooling for about $400 \mathrm{~ms}$, the ions are extracted from the first trap as a pulse and guided into the fringe field of the magnet of the second trap in such a way that the radial energy is negligible. The ion bunch is captured $^{15}$ off center in this trap in order to provide an initial magnetron motion. The ions are subjected to the RF field and then ejected from the trap. The time of flight

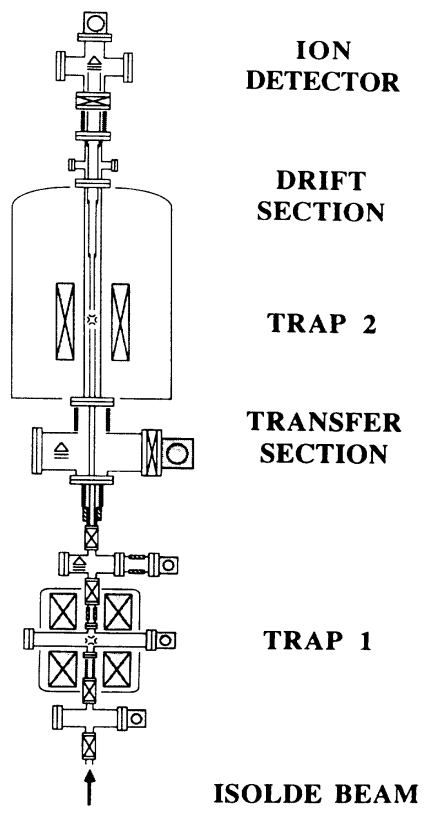

FIG. 1. Experimental setup for direct mass measurements of short-lived isotopes. to a multichannel-plate (MCP) detector placed on top of the superconducting magnet is measured. In resonance at $v=v_{c}$, the gain in kinetic energy (about $20 \mathrm{eV}$ for $A=100$ ) leads to a reduction of up to $40 \%$ in time of flight. A resonance curve is shown in Fig. 2 for ${ }^{120} \mathrm{Cs}$ $\left(T_{1 / 2}=64 \mathrm{~s}\right)$. This resonance was obtained with an $\mathrm{RF}$ interaction time of $T_{\mathrm{RF}}=1.8 \mathrm{~s}$ resulting in a resonance width of $\Delta v_{c}(\mathrm{FWHM})=0.5 \mathrm{~Hz}$ and a resolving power of $R=1500000$. The measurement took $7 \mathrm{~min}$ with a total of 2000 detected ions. The overall efficiency (ratio of the number of ions detected by the MCP detector and the number of ions implanted into the foil of trap 1) was about $5 \times 10^{-5}$.

The cyclotron resonances were fitted by a Gaussian, yielding a statistical precision of $\delta v_{c}^{\text {stat }} \simeq 0.01 \mathrm{~Hz}$. Repeating the measurements several times, the position of the center of resonance scattered within $\delta v_{c}= \pm 0.07$ $\mathrm{Hz}$. We adopt this value for the accuracy of our $v_{c}$ determination. The measured cyclotron frequencies are converted to mass excesses by taking the cyclotron frequency and the mass excess of ${ }^{133} \mathrm{Cs}$ as reference. They are given in Table I.

In order to check the performance of the system, the cyclotron resonances of ${ }^{85} \mathrm{Rb},{ }^{87} \mathrm{Rb}$, and ${ }^{133} \mathrm{Cs}$ were repeatedly measured. Taking the adopted mass of ${ }^{85} \mathrm{Rb}$ (Ref. 1) for calibrating the magnetic field, the mean value of a large number of $v_{c}$ measurements deviated by $-0.6 \mathrm{keV}$ for ${ }^{87} \mathrm{Rb}$ and $+1.5 \mathrm{keV}$ for ${ }^{133} \mathrm{Cs}$ which is well within the uncertainties given in the 1986 mass tables. ${ }^{1}$ Hence mass-dependent systematic errors can be neglected. Also, within the statistical fluctuations, no dependence could be observed on the number of stored ions.

The only systematic shift of $v_{c}$ observed so far is due to contamination by ions of different mass. Since isobars

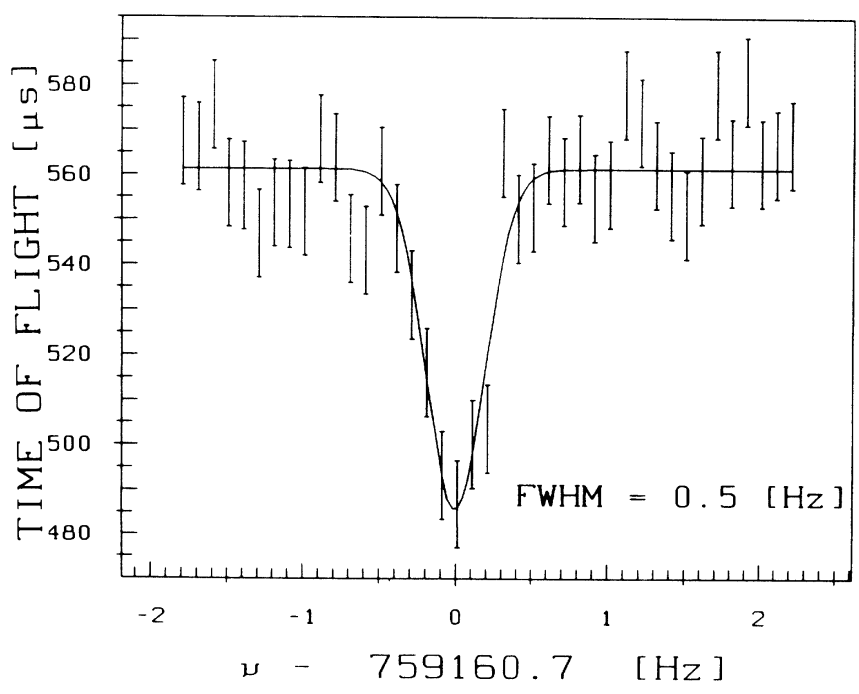

FIG. 2. Cyclotron resonance of ${ }^{120} \mathrm{Cs}\left(T_{1 / 2}=64 \mathrm{~s}\right)$. A resolving power of 1500000 is obtained. 
TABLE I. Cyclotron frequencies and mass excesses of cesium isotopes with mass number $A$ and half-life $T_{1 / 2} .{ }^{133} \mathrm{Cs}$ was taken as reference [mass excess $=-88080(4) \mathrm{keV}$ ]. An isomeric correction was applied in cases where the ground and isomeric states could not be discriminated. The last two columns give the ground-state mass excess as given in the 1986 mass tables and as obtained by a new least-squares adjustment of all mass data available.

\begin{tabular}{|c|c|c|c|c|c|c|c|}
\hline $\begin{array}{c}\text { Isotope } \\
\text { A } \\
\end{array}$ & $\mathrm{T}_{1 / 2}$ & $\begin{array}{c}\text { Cyclotron } \\
\text { Frequency } \\
{[\mathrm{Hz}]}\end{array}$ & $\begin{array}{c}\text { Measured } \\
\text { Mass Excess } \\
{[\mathrm{keV}]}\end{array}$ & $\begin{array}{c}\text { Isomeric } \\
\text { Correction } \\
{[\mathrm{keV}]}\end{array}$ & $\begin{array}{c}\text { Corrected } \\
\text { Mass Excess } \\
{[\mathrm{keV}]}\end{array}$ & $\begin{array}{c}\text { Literature } \\
\text { Mass Excess } \\
{[\mathrm{keV}]}\end{array}$ & $\begin{array}{c}\text { Adjusied } \\
\text { Mass Excess } \\
{[\mathrm{keV}]} \\
\end{array}$ \\
\hline 118 & $16.4 \mathrm{~s}$ & $771998.12(7)$ & $-68409(14)$ & $24(19)$ & $-68433(24)$ & $-68270(130)$ & $-68429(23)$ \\
\hline 119 & $37.7 \mathrm{~s}$ & $765533.55(7)$ & $-72293(15)$ & $33(22)$ & $-72326(27)$ & $-72240(100)$ & $-72342(25)$ \\
\hline 120 & 648 & $759160.87(7)$ & $-73900(15)$ & $24(19)$ & $-73924(24)$ & $-73820(80)$ & $-73900(22)$ \\
\hline 121 & 1368 & $752904.01(7)$ & $-77094(15)$ & $40(20)$ & $-77134(25)$ & $-77110(60)$ & $-77140(23)$ \\
\hline 122 & $21 \mathrm{~s}$ & $746734.94(6)$ & $-78080(15)$ & $7(4)$ & $-78087(17)$ & $-78140(60)$ & $-78086(13)$ \\
\hline $122 \mathrm{~m}$ & $4.5 \mathrm{~min}$ & $746734.49(6)$ & $-78011(15)$ & ? & $-78011(15)$ & & \\
\hline 123 & $5.87 \mathrm{~min}$ & $740678.96(7)$ & $-81049(15)$ & - & $-81049(15)$ & $-81070(40)$ & $-81059(14)$ \\
\hline 124 & $30.8 \mathrm{~s}$ & $734705.96(7)$ & $-81746(15)$ & - & $-81746(15)$ & $-81740(40)$ & $-81744(14)$ \\
\hline 125 & $45 \mathrm{~min}$ & $728838.80(7)$ & $-84083(16)$ & - & $-84083(16)$ & $-84113(17)$ & $-84100(11)$ \\
\hline 126 & $1.64 \mathrm{~min}$ & $723051.89(7)$ & $-84360(16)$ & - & $-84360(16)$ & $-84347(24)$ & $-84354(13)$ \\
\hline 127 & $6.25 \mathrm{~h}$ & $717365.97(6)$ & $-86253(16)$ & - & $-86253(16)$ & $-86243(12)$ & $-86247(9)$ \\
\hline 128 & $3.62 \mathrm{~min}$ & $711755.61(6)$ & $-85941(16)$ & - & $-85941(16)$ & $-85928(6)$ & $-85932(6)$ \\
\hline 129 & $32.06 \mathrm{~h}$ & $706243.38(7)$ & $-87510(17)$ & - & $-87510(17)$ & $-87506(5)$ & $-87507(5)$ \\
\hline 130 & $29.2 \mathrm{~min}$ & $700803.31(6)$ & $-86910(16)$ & - & $-86910(16)$ & $-86853(8)$ & $-86896(10)$ \\
\hline 131 & $9.69 \mathrm{~d}$ & $695456.51(7)$ & $-88081(17)$ & - & $-88081(17)$ & $-88076(6)$ & $-88069(6)$ \\
\hline 132 & $6.475 \mathrm{~d}$ & $690178.92(7)$ & $-87158(17)$ & - & $-87158(17)$ & $-87171(5)$ & $-87165(4)$ \\
\hline 133 & stable & $684991.03(6)$ & reference & - & reference & $-88086(5)$ & $-88080(4)$ \\
\hline 134 & $2.062 \mathrm{y}$ & $679869.03(7)$ & $-86889(17)$ & - & $-86889(17)$ & $-86906(5)$ & $-86901(4)$ \\
\hline 135 & $3 \times 10^{8} y$ & $674833.11(6)$ & $-87568(17)$ & - & $-87568(17)$ & $-87662(7)$ & $-87581(15)$ \\
\hline 136 & $13.16 \mathrm{~d}$ & $669861.28(7)$ & $-86364(18)$ & - & $-86364(18)$ & $-86354(5)$ & $-86348(5)$ \\
\hline 137 & $30.17 \mathrm{y}$ & $664969.50(7)$ & $-86566(18)$ & - & $-86566(18)$ & $-86556(5)$ & $-86552(4)$ \\
\hline
\end{tabular}

a'Isomeric state with longer half-life than ground state.

of cesium are not delivered by ISOLDE and isotopic impurities are eliminated by the mass-selective cooling in trap 1, only contamination with isomeric states of Cs needs to be considered. We found that, in the case of a small energy difference between the ground and isomeric states, only one cyclotron resonance is observed. It appears that, due to the Coulomb interaction between the typically 100 ions stored in the trap, only the cyclotron motion of the center of gravity is excited as was observed before for the axial motion in a Paul trap. ${ }^{16}$ Under this assumption it can be concluded that the ${ }^{122} \mathrm{Cs}$ nuclear state with $T_{1 / 2}=21 \mathrm{~s}$ is the ground state. This follows from the direction of the resonance shift in dependence of the delay between measurement and collection. Combining the mass excesses for ${ }^{122} \mathrm{Cs}^{m}$ and the mixture ${ }^{122} \mathrm{Cs}^{122} \mathrm{Cs}^{m}$ with the ratio of the production yield $(0.1 \pm 0.05)$, one can calculate the ground-state mass of ${ }^{122}$ Cs. The excitation energy of the isomer with $T_{1 / 2}$ $=4.5 \mathrm{~min}$ then becomes $76(24) \mathrm{keV}$. This is the first time that an isomeric excitation energy could be determined by a mass spectrometer.

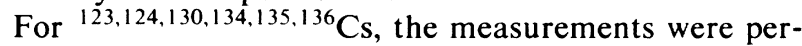
formed with a delay of several isomeric half-lives to let the isomer decay away. In the cases of similar half-lives of ground and isomeric states $\left({ }^{118} \mathrm{Cs}-{ }^{121} \mathrm{Cs}\right)$, the known ratio of the production yields together with excitation energies ${ }^{17}$ can be used to correct for the shift (column 5 of Table I). The last column in Table I shows the mass ex- cess as obtained by a new least-squares adjustment taking into account the data of the present work. The accuracy of the mass data of the isotopes lighter than ${ }^{125} \mathrm{Cs}$ is

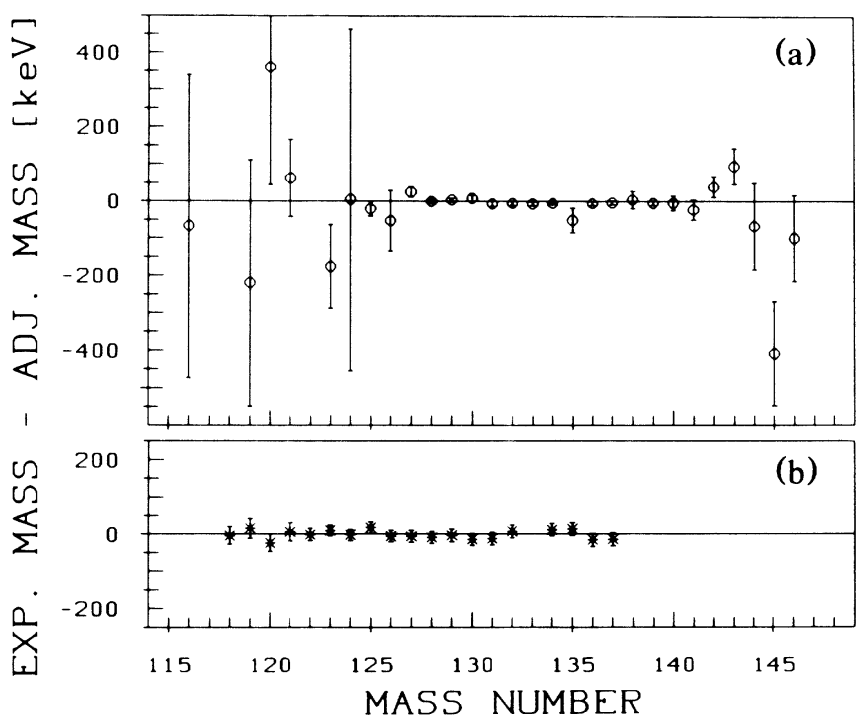

FIG. 3. Mass data of cesium isotopes. The zero line is the result of an adjustment of all data available. The deviations from these adjusted values are plotted for (a) the available $Q_{\beta}$ and reaction data, and (b) the data of the present work by use of the Penning-trap mass spectrometer with ${ }^{133} \mathrm{Cs}$ as reference mass. 


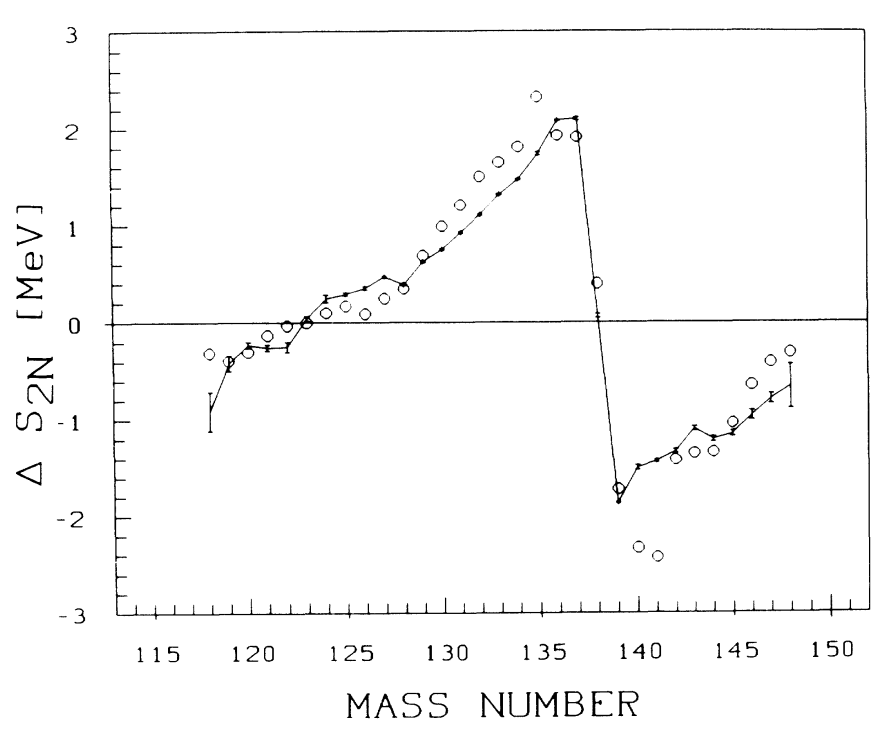

FIG. 4. Two-neutron separation energies of Cs isotopes vs mass number. Experimental values (connected by a line) are compared to a macroscopic-microscopic calculation (open circles) (Ref. 22). The macroscopic part for spherical shape is subtracted.

seen to be considerably increased by the present work.

Our mass excesses agree quite well with the data of the 1986 mass tables except for the case of ${ }^{135} \mathrm{Cs}$ and ${ }^{130} \mathrm{Cs}$. The $6 \sigma$ deviation for ${ }^{135} \mathrm{Cs}$ is entirely due to the $Q_{\beta}$ value of ${ }^{135} \mathrm{Cs}$ reported only as a conference abstract. ${ }^{18}$ This value was therefore rejected in our new adjustment. The $3 \sigma$ deviation for ${ }^{130} \mathrm{Cs}$ is due to the error of $4 \mathrm{keV}$ for the $Q_{\beta}\left({ }^{130} \mathrm{Cs}\right)$ value ${ }^{19}$ assumed in the 1986 mass adjustment. The discrepancy is completely resolved by assigning an error of $50 \mathrm{keV}$. Very recently, the trap measurement was repeated, yielding the same result.

A comparison between this adjustment and the $Q_{\beta}$ and reaction data and our data is shown in Fig. 3. The excellent agreement of the Penning-trap results with the $Q_{\beta}$ and reaction data for nuclei near stability demonstrates the reliability of the Penning-trap technique. Its most outstanding feature is the very high and constant accuracy maintained even for isotopes far from stability. This high accuracy serves to resolve the discrepancy between the Orsay data obtained by a Mattauch-Herzog spectrometer $^{20}$ and $Q_{\beta}$ data on the very light Cs isotopes as shown in Fig. 6 of Ref. 20.

The experimental information on the ground-state properties [spins, magnetic moments, spectroscopic quadrupole moments, changes in charge radii, and deformation parameters for ${ }^{118} \mathrm{Cs}-{ }^{146} \mathrm{Cs}$ (Ref. 21)] result in a rather consistent picture of the Cs isotopes. As an exam- ple, Fig. 4 shows the experimental two-neutron separation energies (connected by a line) together with the results of a macroscopic-microscopic calculation (open circles). ${ }^{22}$ The macroscopic part of the model is subtracted from these values to display the microscopic corrections clearly, e.g., the shell closure at $N=82$. It is seen that the general trend of the experimental $S_{2 N}$ values is reproduced quite well. The remaining discrepancies, especially the irregularities on the neutron-deficient side, exhibit the complex nuclear structure in this region. It is a challenge for theory to meet the new experimental accuracy achievable in mass determination of nuclei far from stability.

This work has been funded by the German Federal Minister for Research and Technology (BMFT) under Contract No. $06 \mathrm{Mz}-188-\mathrm{I}$ and the Natural Sciences and Engineering Research Council.

${ }^{1}$ A. H. Wapstra, G. Audi, and R. Hoekstra, At. Data Nucl. Data Tables 39, 290 (1988).

${ }^{2}$ P. G. Hansen and B. Jonson, Europhys. Lett. 4, 409 (1987).

${ }^{3}$ C. Thibault et al., Phys. Rev. C 12, 644 (1975).

${ }^{4}$ D. J. Viera et al., Phys. Rev. Lett. 57, 3253 (1986).

${ }^{5}$ A. Gillibert et al., Phys. Lett. B 176, 317 (1986).

${ }^{6}$ B. Franzke, Nucl. Instrum. Methods Phys. Res., Sect. B 24/25, 18 (1987).

${ }^{7}$ Y. Fujita et al., in Proceedings of the Eleventh International Mass Spectrometry Conference, Bordeaux, 1988, edited by P. Longevialle (Heyden, London, 1989), Vol. 11 a, p. 640.

${ }^{8}$ G. Gräff, H. Kalinowsky, and J. Traut, Z. Phys. A 297, 35 (1980).

${ }^{9}$ P. B. Schwinberg, R. S. Van Dyck, Jr., and H. G. Dehmelt, Phys. Lett. 81A, 119 (1981).

${ }^{10} \mathrm{G}$. Gabrielse et al., Phys. Rev. Lett. 63, 1360 (1989).

${ }^{11}$ C. Thibault et al., Nucl. Phys. B (Proc. Suppl.) 8, 454 (1989)

${ }^{12}$ St. Becker et al., Int. J. Mass Spectrom. Ion Proc. (to be published).

${ }^{13}$ L. S. Brown and G. Gabrielse, Rev. Mod. Phys. 58, 233 (1986).

${ }^{14} \mathrm{G}$. Bollen et al., J. Appl. Phys. (to be published).

${ }^{15} \mathrm{H}$. Schnatz et al., Nucl. Instrum. Methods Phys. Res., Sect. A 251, 17 (1986).

${ }^{16}$ K. Jungmann et al., Phys. Rev. A 36, 3451 (1987).

${ }^{17}$ G. Audi et al., Nucl. Phys. A378, 443 (1982).

${ }^{18}$ L. Lidofsky, E. Alperovitch, and C. S. Wu, Phys. Rev. 90, 387 (1953).

${ }^{19}$ A. B. Smith, A. C. G. Mitchell, and R. S. Caird, Phys. Rev. 87, 454 (1952).

${ }^{20}$ G. Audi et al., Nucl. Phys. A449, 491 (1986).

${ }^{21}$ E. W. Otten, in Treatise on Heavy Ion Science 8: Nuclei Far from Stability, edited by D. A. Bromley (Plenum, New York, 1989), p. 515.

${ }^{22}$ P. Möller and J. R. Nix, At. Data Nucl. Data Tables 39, 213 (1988). 


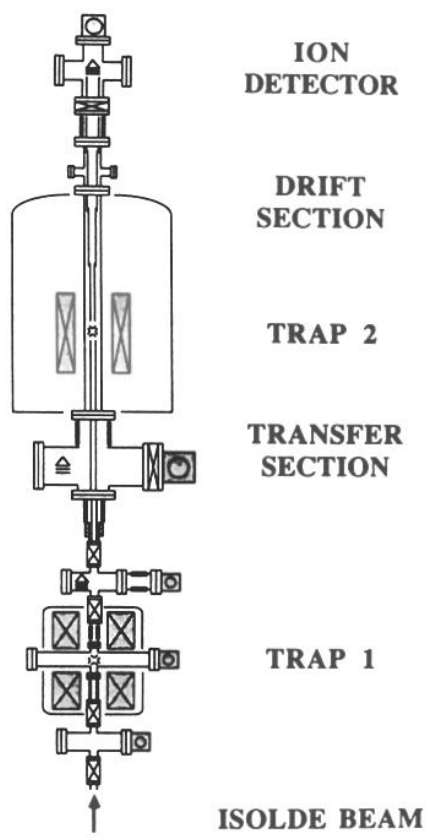

FIG. 1. Experimental setup for direct mass measurements of short-lived isotopes. 